\title{
Management and outcome of pediatric metastatic Wilms' tumor at the National Cancer Institute, Egypt
}

\author{
Moatasem Elayadi ${ }^{1}$, Sarah Magdy ${ }^{1}$, Ehab Khalil ${ }^{2}$ and Wael Zekri ${ }^{\text {1* }}$
}

\begin{abstract}
Background: Wilms' tumor (WT) is the most common renal malignant tumor of childhood. Metastatic WT has a worse prognosis than localized disease. This study aims to assess the clinical outcome and different prognostic factors that influence treatment outcome of pediatric metastatic WT cases treated at National Cancer Institute (NCl), Egypt, between January 2008 and December 2015. Medical records were retrospectively reviewed for clinical, radiological and histopathological data, treatment received, and survival outcome.

Results: In the specified study period, 24/103 (23.3\%) patients with WT were metastatic at presentation. The mean age was $5.25 \pm 2.87$ years (range 2.0-12.7). Abdominal swelling/mass was the commonest presentation (70.8\%). Only 3 patients (12.5\%) had combined lung and liver metastases while 21 patients (87.5\%) had pulmonary-only metastases. All patients had favorable histology tumors with no anaplasia. Nine patients (37.5\%) underwent upfront nephrectomy. Majority of patients (91.7\%) had local stage III disease. Surgical complications were reported in 4 patients; 3 of them had up-front nephrectomy. Only $7 / 21$ patients achieved rapid complete response of pulmonary nodules after 6 weeks of chemotherapy $(\mathrm{CTH})$, and they had a better survival outcome. Patients were followed up till December 2017. Thirteen patients (54.1\%) experienced events during the study period including 5 relapses, 6 cases with disease progression, and 2 patients died out of sepsis. The 3-year event-free and overall survival rates were $48.2 \%$ and $54.2 \%$, respectively.
\end{abstract}

Conclusion: Neo-adjuvant CTH followed by delayed nephrectomy seems more suitable approach in our institute. Pulmonary response to neo-adjuvant CTH appears to be a strong predictor for outcome.

Keywords: Wilms' tumor, Nephroblastoma, Metastatic, Stage IV, Pediatric

\section{Background}

Nephroblastoma or Wilms' tumor (WT) is the most common renal malignant tumor of childhood. Approximately $12 \%$ of WT are metastatic at presentation, with $80 \%$ having pulmonary metastases [1]. The primary distant site for WT metastases is the lungs, while hepatic metastases are much less common [2]. Stage IV disease has the worst outcome between the favorable histology WT and severe short and long-term morbidity due to

\footnotetext{
*Correspondence: waelzekri@gmail.com; waelzekri@cu.edu.eg 'Department of Pediatric Oncology, National Cancer Institute, Cairo University, El-khalig Square, Kasr El-Aini St., Cairo 11796, Egypt Full list of author information is available at the end of the article
}

the more intensive chemotherapy used and also due to the irradiation to both the metastatic sites and the tumor bed [3]. Response of pulmonary nodules to neo-adjuvant chemotherapy $(\mathrm{CTH})$ is an important factor to predict patients' outcome [1]. The purpose of this study is to assess the clinical outcome of WT patients with metastases at initial presentation and to study the different factors that may influence their prognosis.

\section{Methods}

This is a retrospective study, including all newly diagnosed pediatric patients with metastatic unilateral WT from $1^{\text {st }}$ January, 2008 to $31^{\text {st }}$ December, 2015. Records

\section{Springer Open}

() The Author(s). 2020 Open Access This article is licensed under a Creative Commons Attribution 4.0 International License, which permits use, sharing, adaptation, distribution and reproduction in any medium or format, as long as you give appropriate credit to the original author(s) and the source, provide a link to the Creative Commons licence, and indicate if changes were made. The images or other third party material in this article are included in the article's Creative Commons licence, unless indicated otherwise in a credit line to the material. If material is not included in the article's Creative Commons licence and your intended use is not permitted by statutory regulation or exceeds the permitted use, you will need to obtain permission directly from the copyright holder. To view a copy of this licence, visit http://creativecommons.org/licenses/by/4.0/. 
of all patients were reviewed for details of demographic data, initial investigations; CT scans and histopathology, revision of staging, treatment received, and response to treatment.

All patients had CT chest and abdomen with contrast as initial work-up for detection of pulmonary or hepatic metastases. Assessment of treatment response was done after 6 weeks of neo-adjuvant chemotherapy and was discussed through multidisciplinary team (MDT) meetings. All CT films were reviewed by experts in the radiodiagnosis department indicating the metastatic nature of the lung or hepatic lesions. The pathology assessment and determination of favorable vs unfavorable histology followed the guidelines of COG protocol $[4,5]$.

Patients were treated according to modified COG protocol "AREN0533" (Figs. 1 and 2) where they received 3-drug regimen (regimen DD-4A) or 5-drug regimen (regimen $\mathrm{M}$ ). Regimen DD-4A consists of vincristine, doxorubicin, and dactinomycin, while regimen $\mathrm{M}$ consists of the same 3 drugs as DD4A regimen plus cyclophosphamide and etoposide (for doses, refer to Figs. 1 and 2). Unlike the original "AREN0533" protocol, loss of heterozygosity (LOH) status for $1 \mathrm{p}$ and 16q chromosomes was not included in risk stratification for enrolled patients.

Patients were followed up till $31^{\text {st }}$ December 2017. Different prognostic factors were analyzed and correlated with outcome, including the site of metastasis (pulmonary, extra pulmonary, or both), initial management (CTH or upfront nephrectomy), local stage, histology, timing of radiotherapy (RT), and pulmonary response to $\mathrm{CTH}$ in terms of rapid complete response (RCR) or slow incomplete response (SIR).

Overall survival (OS) was defined as the time between date of diagnosis and date of death, from any cause, or the date of last visit. Event-free survival
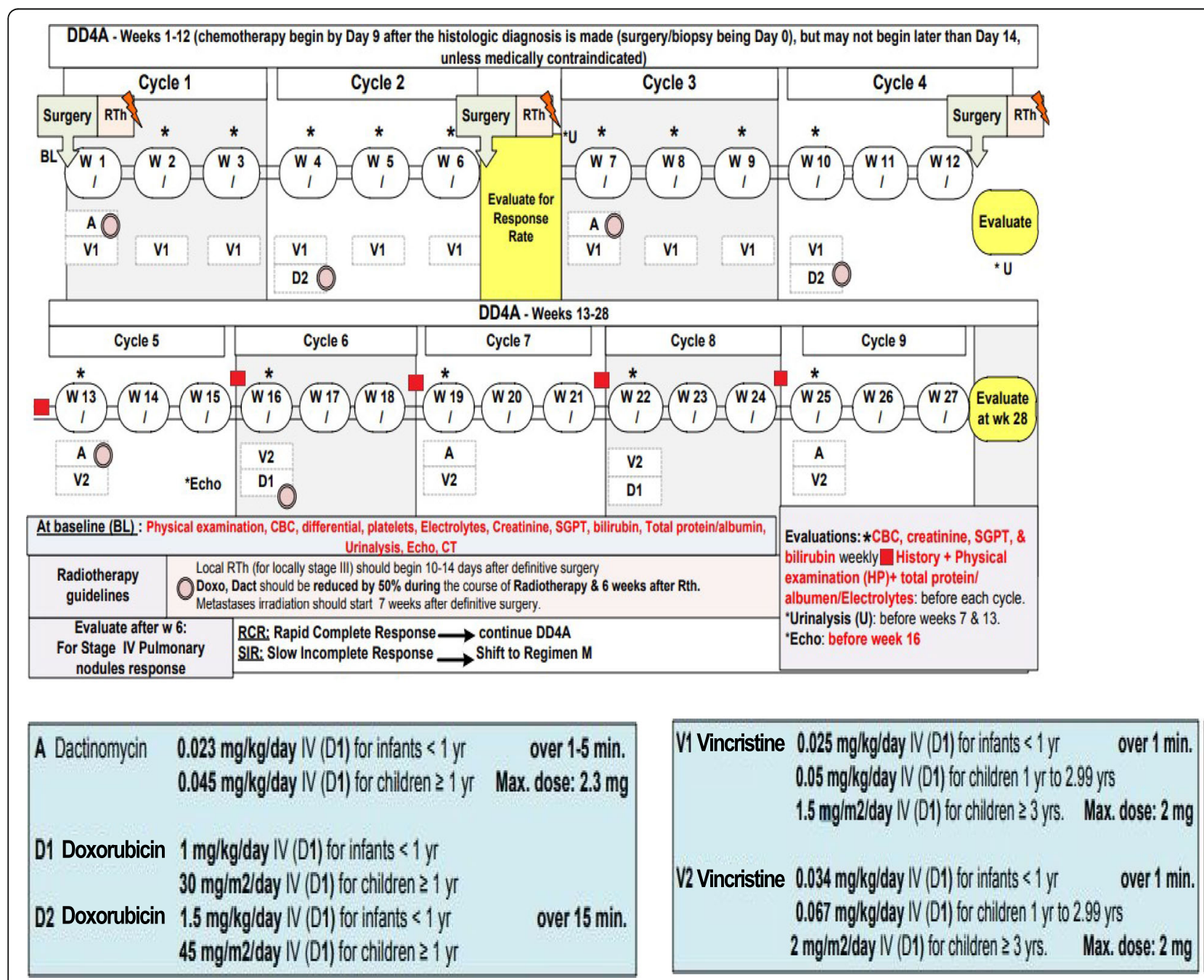

Fig. 1 Regimen DD-4A 


\section{Renal Tumors - Unilateral - Regimen M Unilateral WT- Favorable Histology Stage IV (Pulm. Nodules Slow Response after w 6 DD-4A) Stage IV: Metastases other than pulmonary nodules}
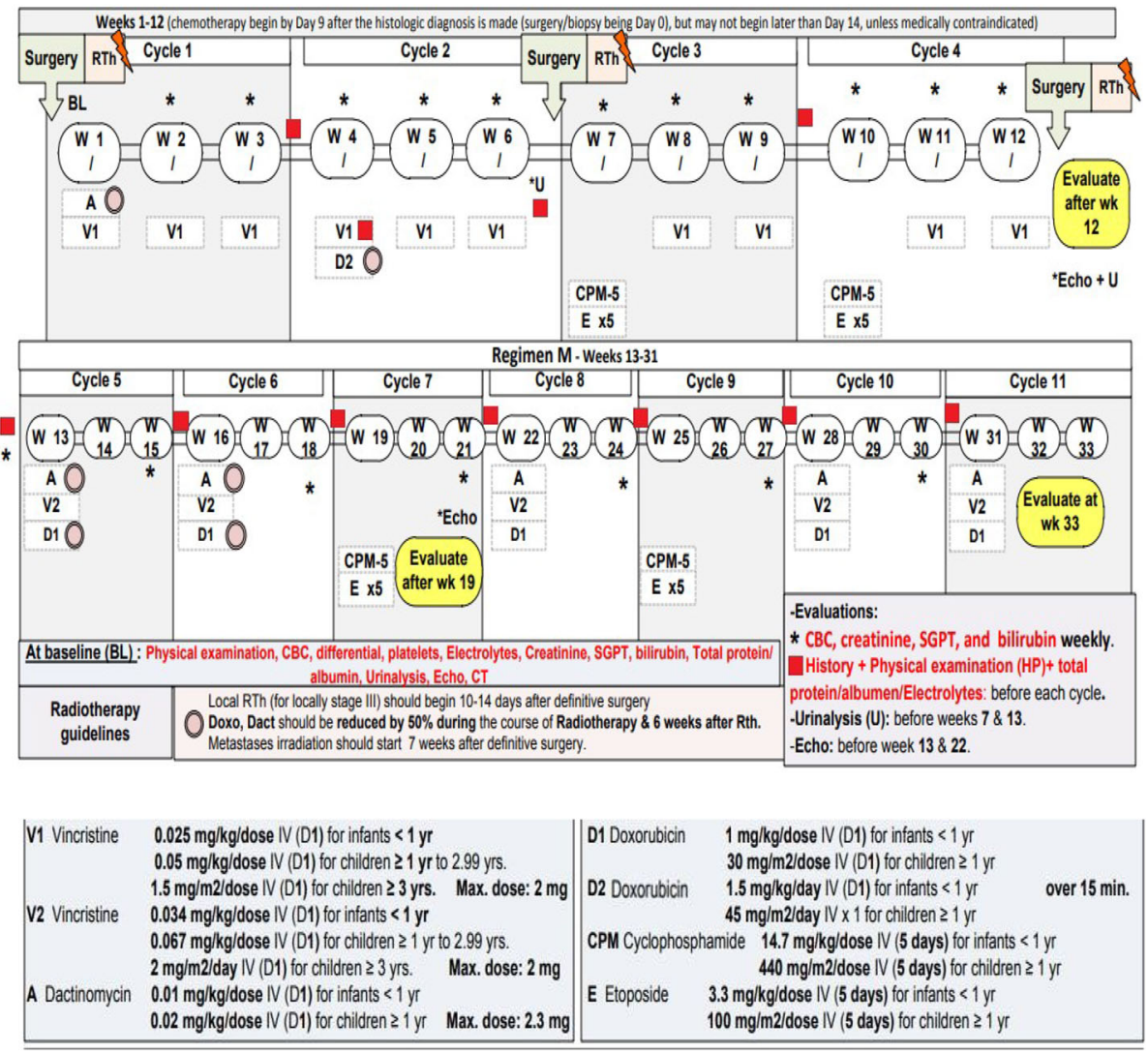

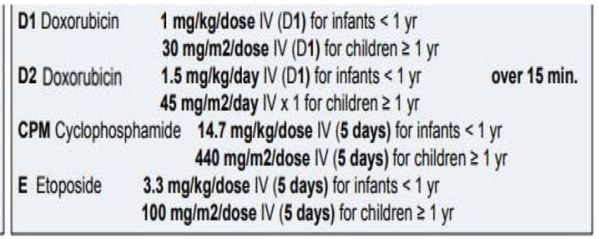

Fig. 2 Regimen $M$

(EFS) was defined as the time from date of diagnosis to date of progression, relapse, death, or last followup, whichever occurred first. Statistical analysis was done using IBM@ SPSS $\odot$ Statistics version 22 (IBM@ Corp., Armonk, NY, USA). Numerical data were expressed as mean and standard deviation or median and range as appropriate. Qualitative data were expressed as frequency and percentage. Survival analysis was done using Kaplan-Meier method, and comparison between two survival curves was done using log-rank test. All tests were two-tailed. A $p$ value < 0.05 was considered significant.

\section{Results}

Patients' characteristics

During the study period, 24 out of 103 patients with WT $(23.3 \%)$ were metastatic at initial presentation to
NCI. The mean age at diagnosis was $5.25 \pm 2.87$ years (range 2.0 to 12.7 years). Females constituted $70.8 \%$ $(17 / 24)$, with a male to female ratio of $1: 2.4$. The primary site of disease was the left kidney in $16 / 24$ patients (66.7\%). Abdominal swelling/mass was the commonest presentation (17 patients, 70.8\%) (Table 1). No congenital anomalies/syndromes were detected in the study patients. All patients were diagnosed to have pulmonary nodules by $\mathrm{CT}$ chest, and only 3 patients (12.5\%) had combined multiple lung and liver metastases at initial presentation.

\section{Initial therapy}

All study patients initially received 3-drug regimen (DD-4A) except for one patient with both lung and liver metastases who received 5-drug regimen (regimen $\mathrm{M})$. All CT abdomen were reviewed initially by 
Table 1 Demographic data and clinical characteristics of the study group $(n=24)$

\begin{tabular}{ll}
\hline Age at diagnosis (years) & \\
\hline Mean \pm SD & $5.25 \pm 2.87$ \\
(Range) & $(2.0-12.7)$ \\
Gender & \\
Male & $7(29.2 \%)$ \\
Female & $17(70.8 \%)$ \\
Laterality of tumor & \\
Right & $8(33.3 \%)$ \\
Left & $16(66.7 \%)$ \\
IVC thrombus & \\
Yes & $3(12.5 \%)$ \\
No & $21(87.5 \%)$ \\
Presenting symptoms & \\
Abdominal swelling & $17(70.8 \%)$ \\
Hematuria & $5(20.8 \%)$ \\
Abdominal pain & $3(12.5 \%)$ \\
Asymptomatic & $1(4.2 \%)$ \\
Site of metastases & \\
Lung & $21(87.5 \%)$ \\
Lung and liver & $3(12.5 \%)$ \\
\hline
\end{tabular}

our surgeons to take the decision either to go for surgery or to start neo-adjuvant chemotherapy. Up-front nephrectomy was done for 9 patients (37.5\%), and 7/9 patients $(77.7 \%)$ had stage III local disease because of presence of post-operative residual $(n=2,28 \%)$, surgical margin positive for infiltration $(n=3,42 \%)$, LN involvement $(n=2,28 \%)$, and intra-operative tumor rupture $(n=1,14 \%)$ (Table 2 and Fig. 3). All remaining patients $(n=15,62.5 \%)$ started neoadjuvant $\mathrm{CTH}$ and showed regressive course of their tumors in CT evaluation post week 6 except for 1 patient whose CT showed a stationary course of the tumor and was shifted to $2^{\text {nd }}$ line CTH.

Twenty-one patients had metastasis in their lungs only, and they were assessed for pulmonary response at week 6. Seven patients (33.3\%) achieved RCR, showing complete resolution of pulmonary nodules by chemotherapy only. There was no surgical intervention for the residual lung nodules after 6 weeks of neo-adjuvant $\mathrm{CTH}$; this is because chest operations are difficult to do together with so much delay of recovery and the possibility of some complications; meanwhile, the revision of the CT chests with our radio-diagnosis departments and whether these residual lesions were at the same sites of the original lesions was the main method of diagnosing rapid vs delayed pulmonary responses.

Surgical complications occurred in 4 patients; 3 patients $(33.0 \%)$ had up-front nephrectomy, and one patient $(6.0 \%)$ underwent delayed nephrectomy after neoadjuvant $\mathrm{CTH}$. These complications were intra-operative tumor rupture $(n=1)$, pulmonary embolism $(n=1)$, and intestinal obstruction $(n=2)$.

All nephrectomy specimens revealed favorable histology with no evidence of anaplasia. Pathology of delayed nephrectomy of 2 patients revealed blastemal predominance. All cases had negative surgical and vascular margins except for 3 patients from the up-front nephrectomy group who had their surgical margins positive for infiltration with tumor cells. None of the 3 patients had early post-operative imaging; however, 2 of them had a gross residual disease in CT evaluation at week 6 (Table 2).

\section{Adjuvant therapy Radiotherapy}

Local RT was given to $21 / 24$ patients (87.5\%). Three patients were deferred from irradiation; one patient had local stage I disease after up-front nephrectomy, and 2

Table 2 Surgical and pathological outcome of patients with up-front nephrectomy $(n=9)$

\begin{tabular}{|c|c|c|c|c|c|c|}
\hline \multirow{2}{*}{$\begin{array}{l}\text { Patient } \\
\text { no. }\end{array}$} & \multirow{2}{*}{$\begin{array}{l}\text { Surgical } \\
\text { complications }\end{array}$} & \multirow{2}{*}{$\begin{array}{l}\text { Post-op } \\
\text { residual }\end{array}$} & \multicolumn{2}{|c|}{ Pathology } & \multirow{2}{*}{$\begin{array}{l}\text { Local } \\
\text { stage }\end{array}$} & \multirow{2}{*}{$\begin{array}{l}\text { Local } \\
\text { residual } \\
\text { @ W (6) }\end{array}$} \\
\hline & & & SM & LNs & & \\
\hline 1 & +ve & Not assessed & +ve & $-v e$ & 3 & $-v e$ \\
\hline 2 & $-v e$ & Not assessed & +ve & Not assessed & 3 & +ve \\
\hline 3 & $-v e$ & Not assessed & + ve & Not assessed & 3 & $+v e$ \\
\hline 4 & $-v e$ & $+v e$ & $-v e$ & $-v e$ & 3 & $-v e$ \\
\hline 5 & $-v e$ & $+v e$ & $-v e$ & Not assessed & 3 & $-v e$ \\
\hline 6 & $-v e$ & $-v e$ & -ve & -ve & 1 & -ve \\
\hline 7 & +ve & $-v e$ & $-v e$ & $+v e$ & 3 & $-v e$ \\
\hline 8 & +ve & $-v e$ & $-v e$ & +ve & 3 & $-v e$ \\
\hline 9 & -ve & -ve & $-v e$ & -ve & 1 & -ve \\
\hline
\end{tabular}




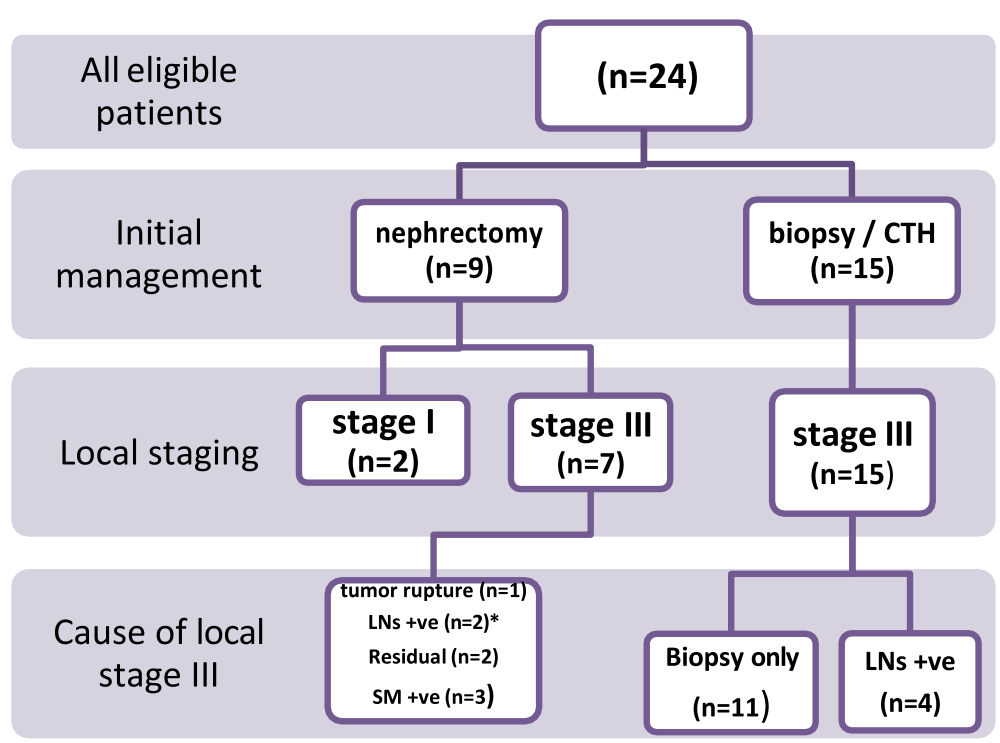

Fig. 3 Initial management and local staging of study patients. CTH, chemotherapy; SM, surgical margin; LNs, lymph nodes. *: including the patient with tumor rupture

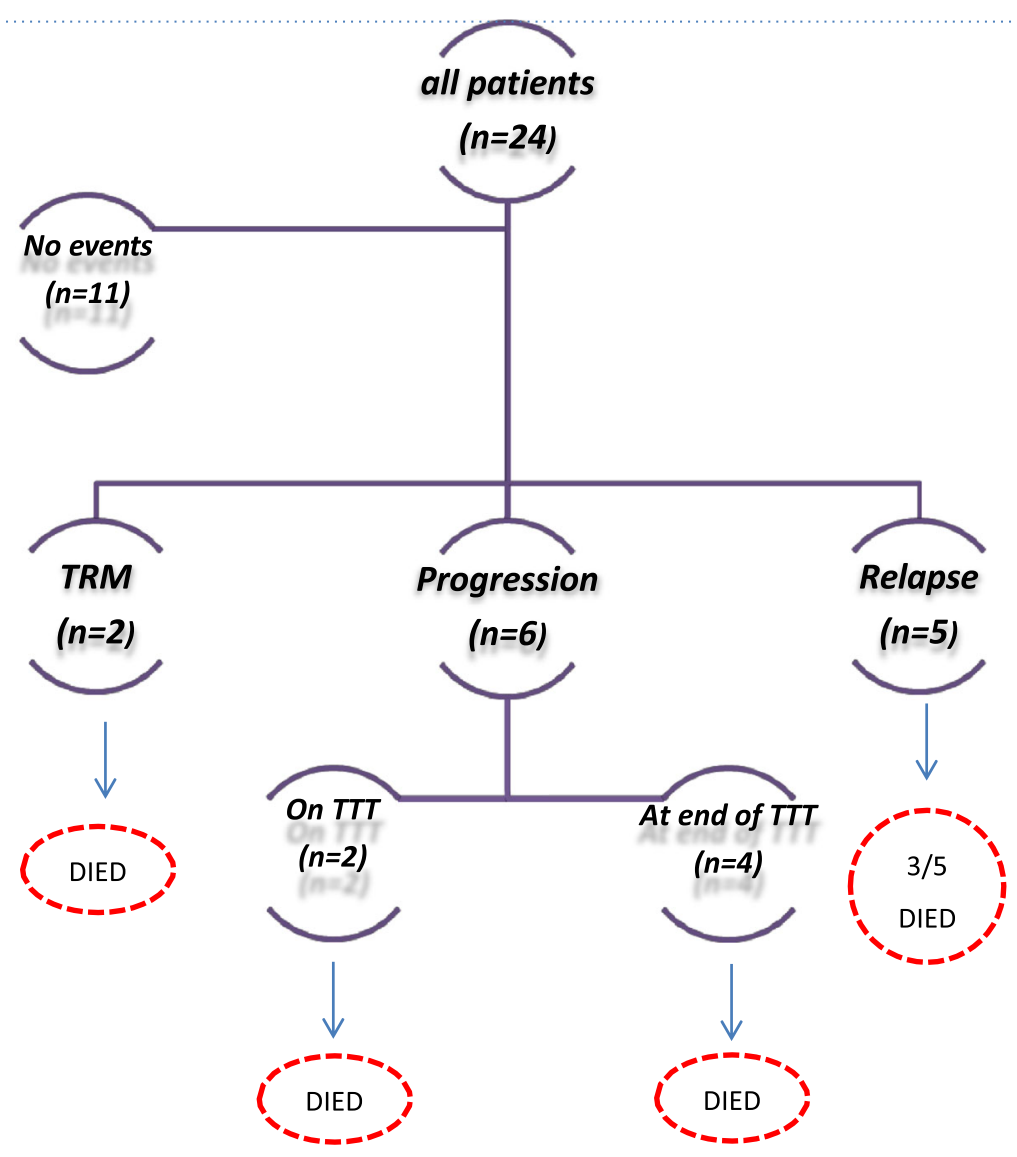

Fig. 4 Events among study cohort. TRM, treatment related mortality; TT, treatment 

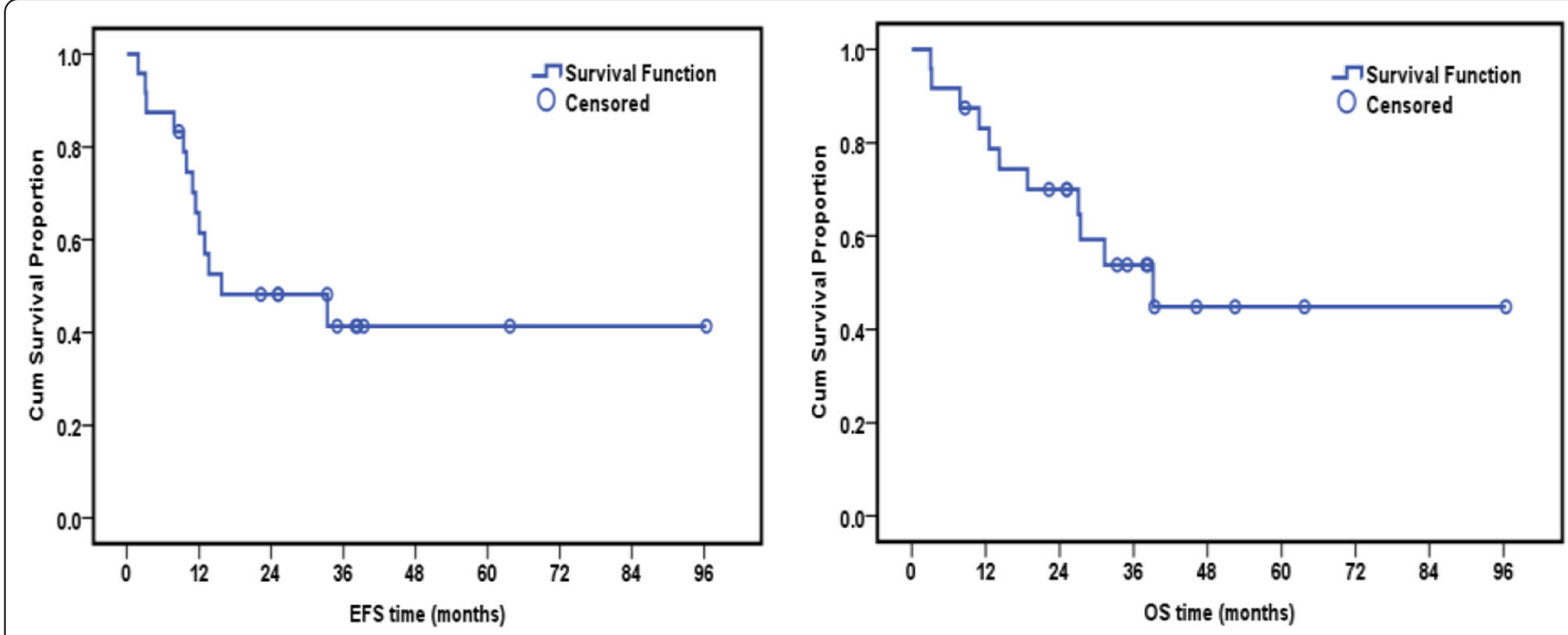

Fig. 5 3-year event-free and overall survival of study patients

patients had progressive disease before local control. Flank RT was implemented to $16 / 21$ patients, and all of them had local stage III. Five patients were treated by whole abdominal irradiation (WAI).

Seven patients achieved pulmonary RCR post neoadjuvant $\mathrm{CTH}$, and $5 / 7$ patients $(71.4 \%)$ were spared lung irradiation. All patients with pulmonary SIR ( $n=$ 14) were treated by lung RT except for 3 patients; one with residual tiny nodule (5 $\mathrm{mm}$ in diameter) and 2 patients developed local progression of their tumors while on treatment. Patients with both lung and liver metastases $(n=3)$ were treated by RT to the lung and liver. For all patients treated with RT, median time between surgery and start of RT was 19 days (range 4-208 days). Only $7 / 21$ patients $(33.3 \%)$ had their RT within 14 days from date of surgery.

\section{Chemotherapy}

Patients with pulmonary RCR $(n=7)$ continued the same 3-drug regimen (DD-4A). Fourteen patients had pulmonary SIR; 10/14 patients were changed to 5-drug regimen, while 4 patients continued the same 3-drug regimen. Three patients had both lung and liver metastases at presentation; one patient was treated by 5-drug regimen, second patient started on 3-drug regimen then was changed to 5-drug regimen after delayed nephrectomy post week 6 while the third patient was treated by 3-drug regimen all through.

\section{Events and survival rates}

Thirteen patients $(54.1 \%)$ experienced events during the study period; 2 patients (15.4\%) had complicated episodes of febrile neutropenia and died out of sepsis while on treatment; 5 patients (38.5\%) had disease relapse, and
6 patients $(46.1 \%)$ had disease progression. Relapse sites were local $(n=1)$, pulmonary $(n=3)$, or both $(n=1)$. Two out of 6 (33.3\%) had local and pulmonary disease progression early on treatment before local control, and $4 / 6$ had progression at end of treatment (Table 4 and Fig. 4).

At a median follow-up of 27.2 months (range from 3.1-96.4 months), the 3-year EFS and 3-year OS were $48.2 \%$ and $54.2 \%$, respectively (Fig. 5). Statistical analysis of survival outcome of study patients in correlation to their demographic and clinical features is summarized in Table 3. EFS and OS for patients who achieved RCR were higher than those who had SIR yet without statistical significance. Similarly, survival rates for patients who received their RTH within 14 days from surgery date were higher than those who received RTH later yet without statistical significance (Tables 3 and 4 and Figs. 6 and 7).

\section{Discussion}

Metastatic WT has a worse prognosis than localized disease and is less frequently seen in developed countries compared to developing world [6]. This study included 24 metastatic WT patients, constituting $23.3 \%$ of all WT patients presented to NCI, Egypt, during the study period. This percentage is similar to that reported in a similar period at Children Cancer Hospital of Egypt (CCHE) which reported $26 \%$ of their patients with WT as stage IV [7]. Reports from other developing African countries indicate a higher incidence of stage IV WT among their cohorts, with a reported incidence of 30.9\%, $40 \%$, and $42 \%$ in Nigeria, Kenya, and South Africa, respectively $[8,9]$. While in developed countries, the incidence is relatively lower, with a reported incidence of 
Table 3 Survival outcome of study patients in correlation to their demographic and clinical characteristics

\begin{tabular}{|c|c|c|c|c|c|c|}
\hline Factors & & $N$ & & 3-year EFS & 3-year OS & \\
\hline & & & $\%$ & $P$ value & $\%$ & $P$ value \\
\hline & All & 24 & 48.2 & & 53.8 & \\
\hline \multirow[t]{2}{*}{ Age } & $<5$ years & 15 & 60.0 & 0.065 & 57.1 & 0.633 \\
\hline & $\geq 5$ years & 9 & 25.9 & & 50.8 & \\
\hline \multirow[t]{2}{*}{ Gender } & Male & 7 & 53.6 & 0.827 & 47.6 & 0.705 \\
\hline & Female & 17 & 37.6 & & 56.5 & \\
\hline \multirow[t]{2}{*}{ Initial management } & Biopsy/CTH & 15 & 37.9 & 0.464 & 66.2 & 0.137 \\
\hline & Nephrectomy & 9 & 44.4 & & 44.4 & \\
\hline \multirow[t]{2}{*}{ Initial metastases } & Lung & 21 & 47.5 & & 57.6 & \\
\hline & Lung and liver & 3 & 0 & * & 33.3 & * \\
\hline \multirow[t]{2}{*}{ IVC involvement } & Yes & 3 & 33.3 & & 66.7 & \\
\hline & No & 21 & 45.5 & * & 51.3 & * \\
\hline \multirow[t]{2}{*}{ Path. LNs involvement } & Yes & 6 & 50.0 & & 33.3 & \\
\hline & No & 9 & 19.5 & 0.657 & 34.6 & 0.655 \\
\hline \multirow[t]{2}{*}{ Pulmonary response at W6 } & $\mathrm{RCR}$ & 7 & 68.6 & & 85.7 & \\
\hline & SIR & 14 & 39.0 & 0.143 & 41.3 & 0.067 \\
\hline \multirow[t]{2}{*}{ SRI } & $\leq 14$ days & 7 & 71.4 & & 64.3 & \\
\hline & $>14$ days & 14 & 34.7 & 0.240 & 48.8 & 0.411 \\
\hline
\end{tabular}

*P value could not be calculated due to small number of patients

IVC inferior vena cava, LNs lymph nodes, SRI surgery radiotherapy interval

$12 \%, 14.9 \%$, and $17 \%$ in Children Oncology Group (COG), German study, and International Society of Pediatric Oncology (SIOP), respectively $[10,11]$. This high incidence of advanced stages of WT noticed in developing countries could be attributed to several factors such as lack of awareness among caregivers and healthcare professionals regarding early presentations of WT, limited resources and poor access to health care that lead to late presentations, and advanced disease stage at diagnosis.

By the end of this study follow-up period, the 3-year EFS and OS were $48.2 \%$ and $54.2 \%$, respectively. Comparable results were reported in similar studies-following SIOP guidelines-in South Africa with 5-year EFS and OS of $54.0 \%$ and $58.5 \%$, respectively [12], and 5-year OS of $54.2 \%$ in another study following National Wilms Tumor Study Group (NWTSG) guidelines [13]. Another study conducted in Lebanon including only 9 patients treated for metastatic WT reported 3-year EFS of $55.6 \%$ and a 3 -year OS of $67 \%$ [14]. Of note, the study carried out at CCHE reports a lower 3-year EFS of $18.2 \%$, yet with higher 3 -year OS of $66 \%$. This might reflect a better salvage rates in their cohorts compared to ours [7].

On the other hand, international studies from developed countries report high survival rates for metastatic
WT patients. In an Italian study; 5-year EFS and OS were $72.1 \%$ and $82.5 \%$, respectively [15]. Another study in Japan reported a 5-year OS of 86.7\% [16]. Also, higher rates were reported by COG and SIOP for stage IV WT patients. The SIOP 2001/GPOH trial reports a 5-year EFS and OS of 73\% and 83\%, respectively [17]. Similarly, NWTS-5 study reports a 5-year EFS and OS of $72 \%$ and $84 \%$, respectively [18]. In 2018, a report from the COG AREN0533 study reported a 4-year EFS and OS of 85.4\% and $95.6 \%$ respectively for stage IV WT patients (17).

There is some evidence that stage IV patients with local stage III disease have lower survival rates compared to local stage I and II patients. In SIOP 93-01, metastatic WT patients with local stage III had a significantly lower OS of $68 \%$ compared with local stage I and II patients $(P$ $<.001$ ), and 5-year EFS rates were $82 \%, 83 \%$, and 57\% for patients with local stage I, II, and III disease, respectively $(P<.001)[19]$. Of note, all of our study patients except for 2 patients had local stage III, a fact that might-in part-explain the relatively lower survival rates of those patients.

Seven out of 9 patients (77.7\%) who did an upfront nephrectomy had stage III local disease. This is relatively a high percentage of local stage III among up-front nephrectomy patients when compared to other studies. In UKW-3 trial, the immediate surgery group had $30 \%$ 


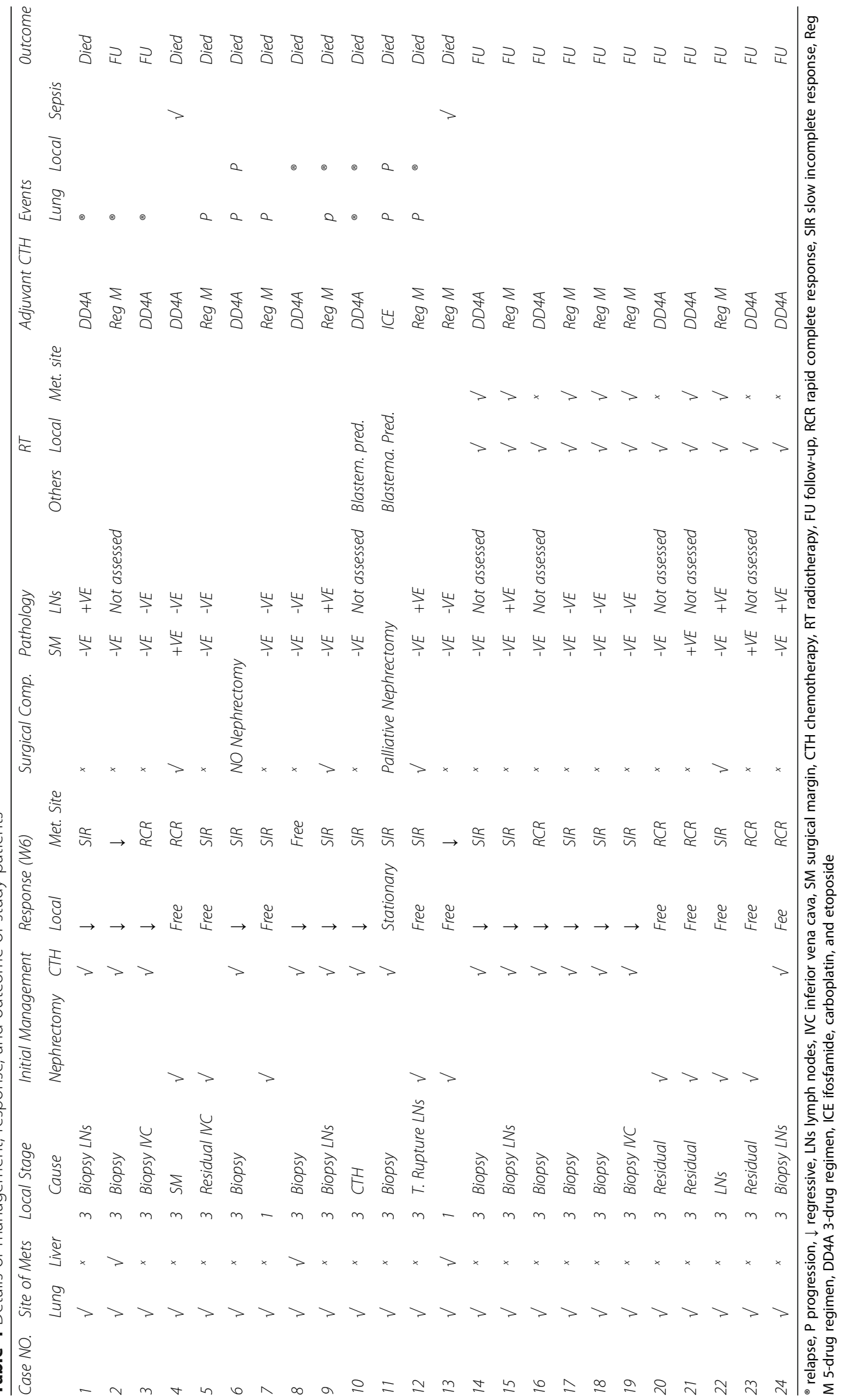



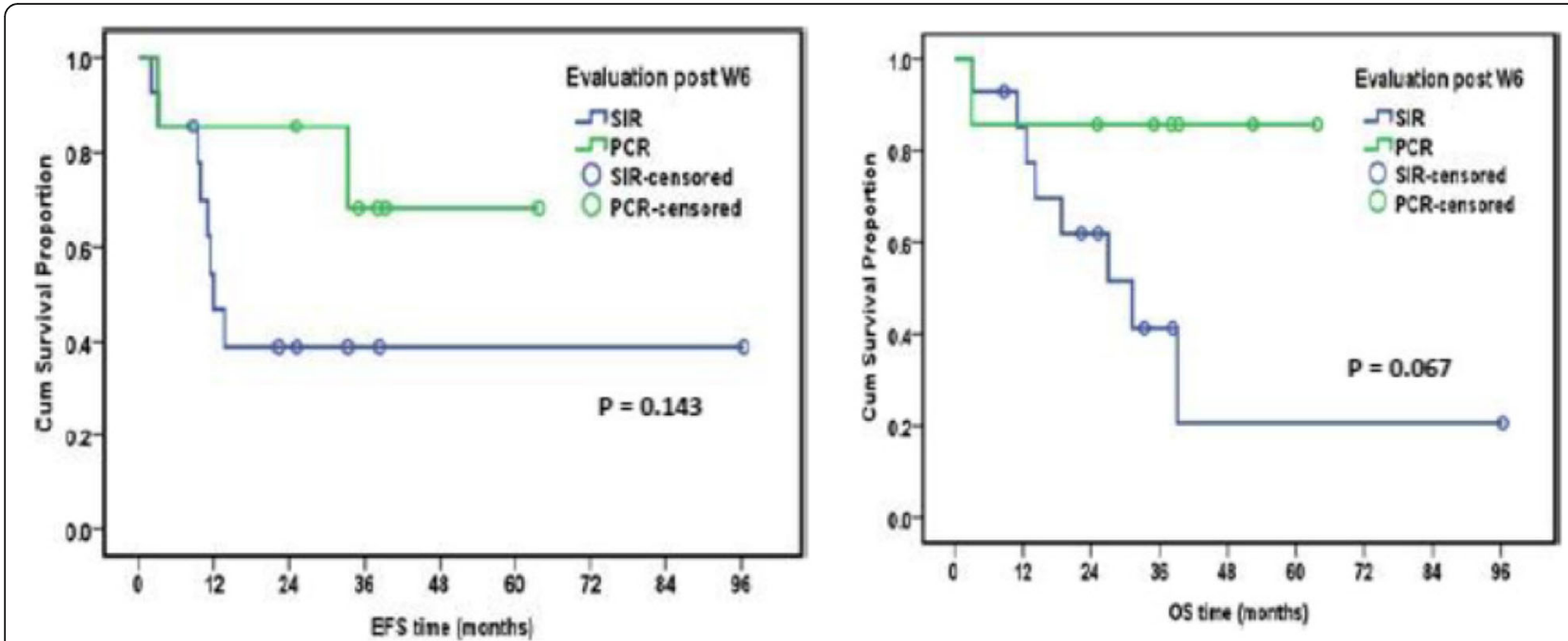

Fig. 6 3-year event-free and overall survival by pulmonary response

of tumors with stage III [20]. Notably, in the CTH and delayed surgery group, only $10 \%$ of tumors were stage III [20]. Moreover, in our study, surgical complications were reported in $3 / 9$ patients (33.3\%) who had up-front nephrectomy in comparison to one patient $(7 \%)$ in the $\mathrm{CTH}$ and delayed nephrectomy group. A study of NWTSG-on 3335 children who underwent primary nephrectomy-reported surgical complications in $12.7 \%$ of patients [21]. The risk of tumor rupture was $15 \%$ in NWTSG for primary surgery and 3\% for chemotherapypretreated cases in SIOP [20]. This was replicated by a randomized study in UK where surgical complications were observed in $14 \%$ of the up-front nephrectomy cohort while none of the neo-adjuvant $\mathrm{CTH}$ pretreated cases experienced any surgical complications [22]. A review of literature for the SIOP trials-since SIOP 1 trial until SIOP 2001-that focuses on the advantages of preoperative chemotherapy reported that surgery-related complications did not exceed $8 \%$ in the pretreated patients [23].

Up-front surgery versus delayed surgery had no statistical significance on outcome, despite high rate of surgical failure upfront. This is because of the poor outcome of the whole group and high rate of progression, and actually there is no evidence that local control can affect outcome of metastatic disease.
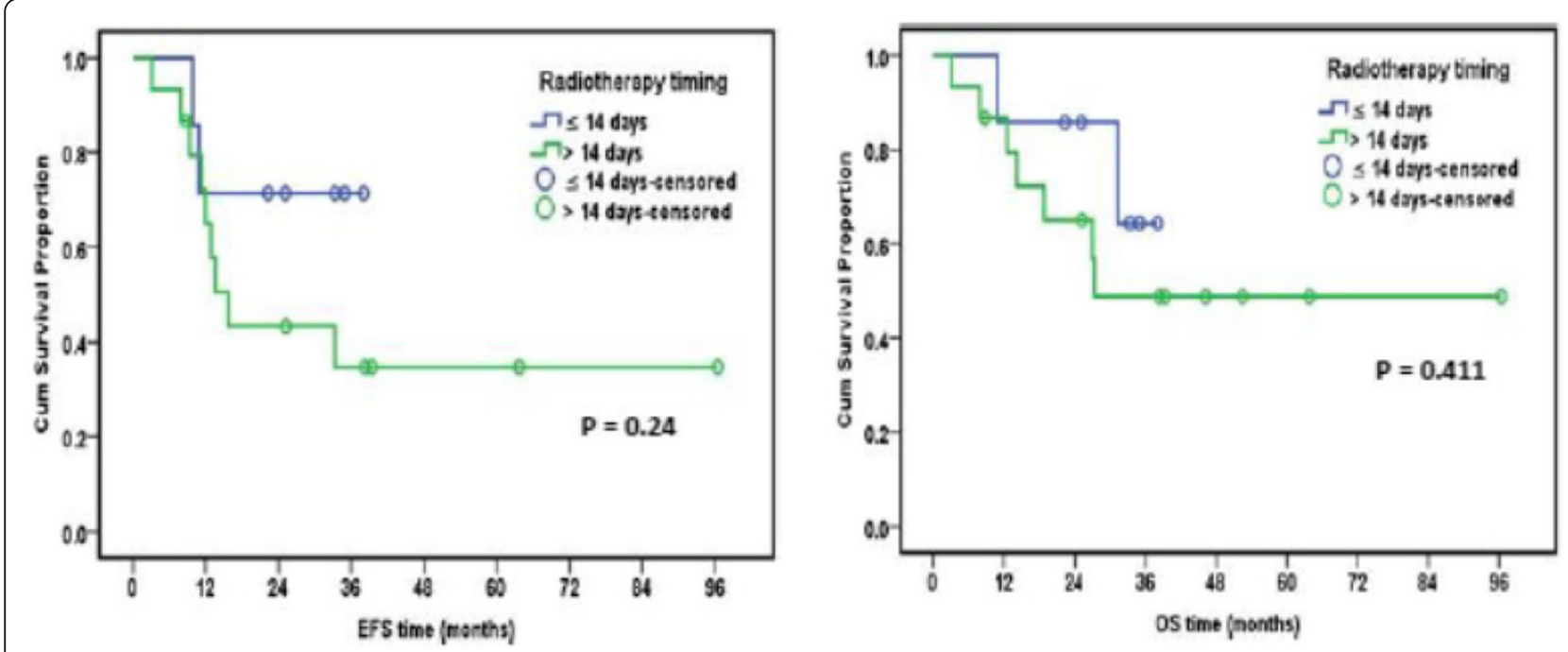

Fig. 7 Event-free and overall survival by surgery radiotherapy interval 


\section{Pulmonary and hepatic response}

The COG, United Kingdom Children's Cancer Study Group (UKCCSG), and SIOP trials evaluated different approaches to the management of children with stage IV pulmonary-only FHWT, seeking to avoid the use of whole-lung irradiation (WLI) because of its significant acute and long-term toxicity [24].

In this cohort, there were 2 cases with treatmentrelated mortality, specifically from severe sepsis. One case was in RCR after week 6 and was on lower doses of chemotherapy (3-drug regimen), while the other patient was on the higher doses of chemotherapy and went into severe neutropenia. Both cases had bacterial growth in their blood cultures together with pulmonary infection. This raises the importance of proper education and counselling for parents and caregivers regarding infection control measures and more importantly regarding early recognition of infectious complications in order to seek medical support when appropriate. Also, there should be close follow-up for the patient condition and their labs to give the proper supportive measures including empirical broad spectrum antibiotics and bone marrow stimulating factors if needed.

In our study, 7 patients (33\%) of 21 patients with pulmonary-only FHWT achieved RCR. The SIOP 93-01 conducted a study on 234 patients with only pulmonary metastases, who received 6 weeks of neo-adjuvant 3drug $\mathrm{CTH}$, followed by nephrectomy. One hundred forty-eight $(67.3 \%)$ of 220 patients who had complete data achieved RCR with combination CTH alone. An additional 37 patients required surgical resection of one or more pulmonary nodules to achieve RCR [18]. In contrast to the SIOP study, COG reported that only 133 of 292 patients $(45.5 \%)$ with only pulmonary metastases were in RCR after 6 weeks of CTH [25], a finding possibly related to the administration of lower cumulative doses of doxorubicin during the pre-nephrectomy $\mathrm{CTH}$ period [18].

In our study, patients with RCR post 6 weeks of CTH had a better survival outcome compared to those with SIR, although without statistical significance. Of course, such results must be carefully interpreted with consideration to the very small number of studied patient and number of events. In the SIOP 93-01 study, 5-year EFS was $77 \%$ for those who achieved complete remission (CR) with CTH only, $84 \%$ for those who required surgical resection of residual nodules to achieve CR, $46 \%$ for patients with inoperable pulmonary metastases treated without WLI, and $50 \%$ for those with inoperable pulmonary metastases treated with WLI [18]. So, surgery should be encouraged for any accessible residual pulmonary nodules after neo-adjuvant CTH. In NWTSG-5, patients with stage IV FHWT with metastases limited to the lung had 5-year EFS of $85 \%$ in the setting of complete lung nodule response (CR) by day 70 versus $74 \%$ with incomplete lung nodule response, with all patients treated with 3-drug regimen [26].

A recent COG study reported that FHWT patients with week 6 lung nodule RCR treated without lung RT had 4-year EFS and OS of 79.5\% and 96.1\%, respectively. While for patients with SIR, 4-year EFS and OS estimates were $88.5 \%$ and $95.4 \%$, respectively, with more intensified CTH (5-drug regimen) together with WLI [25].

As for patients with hepatic metastases, both the NWTS and the SIOP analyses show that patients with residual liver disease after treatment with $\mathrm{CTH}$ and/or RT that could be completely resected did well, suggesting that there is a role for complete surgical resection of residual metastases after adjuvant therapy [27].

\section{Local radiotherapy}

As per protocol, irradiation should start at day 10-14 of therapy (with surgery done at day zero). In the current study, the median time to start radiotherapy after surgery was 19 days, with only $7 / 21$ patients (33.3\%) started their RT within 14 days from surgery date. This delayin most cases-resulted from the burden of large numbers of patients relative to the capacity of RT machines. The National Cancer Database (NCDB) reports that for non-metastatic patients, surgery to radiotherapy interval $\leq 14$ days correlates with improved overall survival. However, no association was noted for patients with metastases [28].

\section{Conclusions}

The incidence of stage IV disease at presentation among our patients with WT is relatively higher than international groups, yet it is remarkably lower than other developing countries. This may reflect the variation in levels of awareness among caregivers and healthcare professionals as well as proportion of patients with delayed presentations rather than true ethnic variability. Abundance of local stage III disease among our patients, non-compliance to treatment protocols, and delay in starting irradiation might contribute to the relatively poorer outcome for our patients with metastatic WT compared to similar patients in other developed countries. Neo-adjuvant CTH and delayed nephrectomy approach is more suitable practice in the setup of a limited-resource center receiving large numbers of patients with advanced stage WT like our institute. Despite lack of statistical significance, the pulmonary response to neo-adjuvant CTH appears to be a strong predictive factor for outcome of patients with only pulmonary metastasis, and this needs further validation on a larger number of cases. 


\section{Limitations of the study}

This study was conducted retrospectively, and hence we were unable to justify the rational for some deviations from the therapy protocol. Some decisions related to field planning of RTH or upgrading treatment for slow responders were not complying with the protocol, and no clear explanation could be retrieved from patients' records. Some fine details were difficult to verify in few cases as well, such as the reasons of delay in surgery or in starting RTH for some patients. The relatively small number of study patients precluded proper statistical inference for many studied variables.

\begin{abstract}
Abbreviations
CCHE: Children Cancer Hospital, Egypt; COG: Children's Oncology Group; CR: Complete remission; CT: Computed tomography; CTH: Chemotherapy; EFS: Event-free survival; FHWT: Favorable histology Wilms' tumor; GPOH: German Paediatric Oncology and Haematology Group; LOH: Loss of heterozygosity; NCDB: National Cancer Database; NCl: National Cancer Institute; NWTSG: National Wilms' Tumor Study Group; OS: Overall survival; RCR: Rapid complete response; RT: Radiotherapy; SIOP: International Society of Paediatric Oncology; SIR: Slow incomplete response; UKCCSG: United Kingdom Children's Cancer Study Group; WAl: Whole abdominal irradiation; WLI: Whole lung irradiation; WT: Wilms' tumor
\end{abstract}

\section{Acknowledgements}

Not applicable

\section{Authors' contributions}

Study concept: WZ. Study design: WZ, ME. Data acquisition: SM. Data analysis and interpretation: SM, WZ, ME, and EKh. Manuscript preparation: SM, WZ, ME, and EKh. Manuscript editing: WZ, ME, and EKh. Manuscript review: WZ. All authors have read and approved the manuscript.

\section{Authors' information}

Not applicable

\section{Funding}

There was no funding for this study by any means.

\section{Availability of data and materials}

The datasets used and analyzed during the current study are available from the corresponding author on reasonable request.

\section{Ethics approval and consent to participate}

Institutional Review board (IRB) at the National Cancer Institute, Cairo University has approved the waiver of patient consent form because of the retrospective nature of the study which used archived data and did not affect patients' well-being in any way. The reference number is not applicable.

\section{Consent for publication}

Not applicable

\section{Competing interests}

The authors declare that the research was conducted in the absence of any commercial or financial relationships that could be construed as a potential conflict of interest.

\section{Author details}

${ }^{1}$ Department of Pediatric Oncology, National Cancer Institute, Cairo University, El-khalig Square, Kasr El-Aini St., Cairo 11796, Egypt. ${ }^{2}$ Department of Radiation Oncology, National Cancer Institute, Cairo University, Cairo, Egypt.
Received: 12 October 2019 Accepted: 30 March 2020

Published online: 15 April 2020

\section{References}

1. Davidoff AM. Wilms tumor. Adv Pediatr Infect Dis. 2012;59(1):247-67.

2. Davidoff AM. Wilms' tumor. Curr Opin Pediatr. 2009;21(3):357-64.

3. Szychot E, Apps J, Pritchard-Jones K. Wilms' tumor: biology, diagnosis and treatment. Transl Pediatr. 2014;3(1):12-24.

4. Murphy WM, Grignon DJ, Perlman EJ: Tumors of the kidney, bladder and related urinary structures. fourth, 2004.

5. Zuppan CW, Beckwith JB, Luckey DW. Anaplasia in unilateral Wilms tumor. A report from the National Wilms Tumor Study Pathology Center. Hum Pathol. 1988:19:1199-209.

6. John R, Kurian JJ, Sen S, Gupta MK, Jehangir S, Mathew LG, et al. Clinical outcomes of children with Wilms tumor treated on a SIOP WT 2001 protocol in a tertiary care hospital in south India. J Pediatr Urol. 2018 Dec; 14(6):547.e1-7.

7. El-Ayadi M, Zekri W, Zaghloul M, Younes A, El Desouky E, Ebeid E. Clinical characteristics and treatment outcomes of unilateral Wilms' tumor in Egypt, report from a prospective cohort analysis. Poster presentation at SIOP Conference, 2014. https://www.researchgate.net/publication/274951514.

8. Ekenze S, Aguqua-Obianyo NEN, Odetunde OA. The challenge of nephroblastoma in a developing country. Ann Oncol. 2006;17(10):1598-600.

9. Njuguna F, Martijn HA, Kuremu RT, Saula P, Kirtika P, Olbara G, et al. Wilms tumor treatment outcomes: perspectives from a low-income setting. J Glob Oncol. 2017:3(5):555-62.

10. Berger M, Fernandez-Pineda I, Cabello R, Ramírez-Villar GL, Márquez-Vega C, Nustede $\mathrm{R}$, et al. The relationship between the site of metastases and outcome in children with stage IV Wilms tumor. J Pediatr Hematol Oncol. 2013 Oct;35(7):518-24.

11. van den Heuvel-Eibrink MM, Hol JA, Pritchard-Jones $\mathrm{K}$, van Tinteren $\mathrm{H}_{\text {, }}$ Furtwängler $\mathrm{R}$, Verschuur $\mathrm{AC}$, et al. Position paper: rationale for the treatment of Wilms tumour in the UMBRELLA SIOP-RTSG 2016 protocol. Nat Rev Urol. 2017;14(12):743-52.

12. Aronson DC, Maharaj A, Sheik-Gafoor MH, Hadley GP. The results of treatment of children with metastatic Wilms tumours (WT) in an African setting: do liver metastases have a negative impact on survival? Pediatr Blood Cancer. 2012 Aug;59(2):391-4.

13. Davidson A, Hartley P, Desai F, Daubenton J, Rode H, Millar A. Wilms tumour experience in a South African centre. Pediatr Blood Cancer. 2006 Apr:46(4):465-71.

14. Rabeh W, Akel S, Eid T, Muwakkit S, Abboud M, El Solh H, et al. Wilms tumor: successes and challenges in management outside of cooperative clinical trials. Hematol Oncol Stem Cell Ther. 2016 Mar 1:9(1):20-5.

15. Spreafico F, Biasoni D, Lo Vullo S, Gandola L, D'Angelo P, Terenziani M, et al. Results of the third AIEOP cooperative protocol on Wilms tumor (TW2003) and related considerations. J Urol. 2017 Nov; 198(5):1138-45.

16. Oue T, Fukuzawa M, Koshinaga T, Okita H, Nozaki M, Chin M, et al. Management of pediatric renal tumor: past and future trials of the Japan Wilms Tumor Study Group. Pediatr Int. 2015;57(5):828-31.

17. Warmann SW, Furtwängler R, Blumenstock G, Armeanu S, Nourkami N, Leuschner I, et al. Tumor biology influences the prognosis of nephroblastoma patients with primary pulmonary metastases. Ann Surg 2011;254(1):155-62.

18. Green DM. Considerations in the diagnosis and management of pediatric patients with favorable histology Wilms tumor who present with only pulmonary nodules. Pediatr Blood Cancer. 2016;63(4):589-92.

19. Verschuur A, Van Tinteren H, Graf N, Bergeron C, Sandstedt B, de Kraker J. Treatment of pulmonary metastases in children with stage IV nephroblastoma with risk-based use of pulmonary radiotherapy. J Clin Oncol. 2012 Oct 1;30(28):3533-9.

20. Bhatnagar S. Management of Wilms' tumor: NWTS vs SIOP. J Indian Assoc Pediatr Surg. 2009 Jan;14(1):6-14

21. Erginel B. Wilms tumor and its management in a surgical aspect. In van den Heuvel-Eibrink MM, editor. Wilms Tumor [Internet]. Brisbane, Australia, Codon Publication, March 2016.

22. Mitchell C, Pritchard-Jones K, Shannon R, Hutton C, Stevens S, Machin D, et al. Immediate nephrectomy versus preoperative chemotherapy in the management of non-metastatic Wilms' tumour: results of a randomised trial (UKW3) by the UK Children's Cancer Study Group. Eur J Cancer. 2006:42(15): 2554-62. 
23. Godzinski J. The current status of treatment of Wilms' tumor as per the SIOP trials. J Indian Assoc Pediatr Surg. 2015 Jan;20(1):16-20.

24. Heaton TE, Davidoff AM. Surgical treatment of pulmonary metastases in pediatric solid tumors. Semin Pediatr Surg. 2016 Oct;25(5):311-7.

25. Dix DB, Seibel NL, Chi Y-Y, Khanna G, Gratias E, Anderson JR, et al. Treatment of stage IV favorable histology Wilms tumor with lung metastases: a report from the Children's Oncology Group AREN0533 study. J Clin Oncol. 2018;36(16):1564-70.

26. Dix DB, Gratias EJ, Seibel N, Anderson JR, Mullen EA, Geller Jl, et al. Omission of lung radiation in patients with stage IV favorable histology Wilms tumor (FHWT) showing complete lung nodule response after chemotherapy: a report from Children's Oncology Group study AREN0533. J Clin Oncol 2015; 33(15_Suppl): pp 10011.

27. Ehrlich PF, Ferrer FA, Ritchey ML, Anderson JR, Green DM, Grundy PE, et al. Hepatic metastasis at diagnosis in patients with Wilms tumor is not an independent adverse prognostic factor for stage IV Wilms tumor. Ann Surg. 2009;250(4):642-8.

28. Stokes CL, Stokes WA, Kalapurakal JA, Paulino AC, Cost NG, Cost CR, et al. Timing of radiation therapy in pediatric Wilms tumor: a report from the National Cancer Database. Int J Radiat Oncol Biol Phys. 2018;101(2):453-61.

\section{Publisher's Note}

Springer Nature remains neutral with regard to jurisdictional claims in published maps and institutional affiliations.

\section{Submit your manuscript to a SpringerOpen ${ }^{\circ}$ journal and benefit from:}

- Convenient online submission

- Rigorous peer review

- Open access: articles freely available online

- High visibility within the field

- Retaining the copyright to your article 